\section{Evaluación de la posición condilar mediante tomografía computarizada de haz cónico en pacientes Clase III sometidos a cirugía ortognática}

\section{Evaluation of the condylar position by conical beam computed tomography in Class III patients undergoing orthognathic surgery}

\begin{abstract}
Resumen
Objetivo. El propósito de este estudio fue comparar los cambios mediante tomografía computarizada de haz cónico en el posicionamiento condilar de pacientes Clase III antes y después de una osteotomía sagital bilateral de rama mandibular indicada para la retroposición mandibular. Métodos. Se analizaron 30 pacientes, 16 mujeres y 14 hombres con rango de edad de 15 a 40 años y deformidad dentofacial Clase III no tratada que asistieron a consulta de diagnóstico en el periodo del año 2013 al 2016 en el Hospital Regional "General Ignacio Zaragoza" (CDMX, México), realizándoseles mediciones de la posición condilar en tres tiempos: prequirúrgico, intermedio (4 días posquirúrgico) y final ( 9 meses posquirúrgico), en dos planos: corte sagital y corte coronal. Resultados. No se observó diferencia significativa en los espacios anterior, central y posterior antes $(2,56 \pm 0,55 \mathrm{~mm} ; 1,78 \pm 0,48 \mathrm{~mm} ; 1,92 \pm 0,36 \mathrm{~mm})$ y después $(2,68 \pm 0,51 \mathrm{~mm} ; 1,87 \pm$ $0,43 \mathrm{~mm} ; 2,01 \pm 0,37 \mathrm{~mm})$, diferencia de medias $-0,120 ;-0,085 ;-0,090 p=0,921 ; 0,948$ y 0,778 , respectivamente. Del mismo modo, en el corte coronal no se observan cambios significativos en los ángulos condilares derecho antes $\left(68,25 \pm 1,56^{\circ}\right)$ y después $(68,77$ $\left.\pm 1,63^{\circ}\right) p=0,217$; e izquierdo antes $\left(68,92 \pm 1,63^{\circ}\right)$ y después $\left(69,30 \pm 2^{\circ}\right) p=0,215$. Conclusiones. La osteotomía sagital bilateral de rama mandibular en pacientes Clase III es una técnica quirúrgica que ofrece mínimas alteraciones condilares, pues mantiene una estabilidad condilar en el posoperatorio a 9 meses.
\end{abstract}

Palabras clave: Cóndilo mandibular; Tomografía computarizada de haz cónico; Procedimientos quirúrgicos ortognáticos; Prognatismo (fuente: DeCS BIREME).

\begin{abstract}
Objective. The purpose of this study was to compare the changes by conical beam computed tomography in the condylar positioning of Class III patients before and after a sagittal osteotomy of the bilateral mandibular ramus in Class III indicated for mandibular retroposition. Methods. Thirty patients were analyzed, 16 women and 14 men with an age range of 15 to 40 years and untreated Class III dentofacial deformity who were attended by diagnostic consultation during the period from 2013 to 2016 at the "Gen-
\end{abstract}

\section{Artículo Original}

José Ernesto Miranda Villasana ${ }^{1, a}$, Sergio Esquivel-Martin ${ }^{1, b}$, Edgar García-Torres ${ }^{2, c}$, Oscar Eduardo Almeda-Ojeda 2,c, Graciela Zambrano-Galván ${ }^{2, d}$, Víctor Hiram Barajas-Pérez ${ }^{2, c}$

${ }^{1}$ Hospital Regional "General Ignacio Zaragoza”, Ciudad de México, México.

${ }^{2}$ Universidad Juárez del Estado de Durango, Facultad de Odontología, Durango, México.

a Maestría en Educación.

${ }^{b}$ Especialista Cirugía Oral y Maxilofacial.

${ }^{c}$ Maestría en Ciencias Estomatológicas.

${ }^{\text {d }}$ Doctora en Ciencias Biomédicas.

\section{Correspondencia:}

Víctor Hiram Barajas-Pérez: hiram.barajas@ujed.mx Calz. Ignacio Zaragoza 1711, Ejército Constitucionalista, Chinam Pac de Juárez, 09220 Ciudad de México, CDMX ORCID: 0000-0002-7182-7393

\section{Coautores:}

José Ernesto Miranda Villasana: ernestomiranda@prodigy.net.mx ORCID: 000-0003-2395-6366

Sergio Esquivel-Martin: sergioesquivelmartin@gmail.com ORCID: 0000-0002-6665-6753

Edgar García-Torres: smiledent.coi@hotmail.com ORCID: 0000-0002-7799-4352

Oscar Eduardo Almeda-Ojeda: oscar.almeda@ujed.mx ORCID: 0000-0001-9885-1644

Graciela Zambrano-Galván: gzambrano@ujed.mx ORCID: 0000-0003-4502-4017

Editor:

Yuri Castro-Rodríguez

Universidad Nacional Mayor de San Marcos, Perú.

Conflicto de intereses: los autores declaramos que no existe ningún tipo de conflictos de interés.

Fuente de financiamiento: el proyecto se lleva a cabo en pacientes derechohabientes del ISSSTE. Los médicos en formación son becarios del mismo instituto.

Recibido: $14 / 10 / 19$

Aceptado: 24/01/20

Publicado: $24 / 02 / 20$

(C) Los autores. Este artículo es publicado por la revista Odontología Sanmarquina de la Facultad de Odontología, Universidad Nacional Mayor de San Marcos. Este es un artículo de acceso abierto, distribuido bajo los términos de la licencia Creative Commons Atribucion - No Comercia_Compartir Igual 4.0 Internacional. (http://creativecommons.org/licenses/by-nc-sa/4.0/) que permite el uso no comercial, distribución y reproducción en cualquier medio, siempre que la obra original sea debidamente citada. 
eral Ignacio Zaragoza" Regional Hospital (CDMX, Mexico), performing measurements of the condylar position in three stages: presurgical, intermediate (4 days after surgery) and final (9 months after surgery), in two planes: sagittal section and coronal section. Results. No significant difference was observed in the anterior, central and posterior spaces before $(2.56 \pm 0.55 \mathrm{~mm} ; 1.78 \pm 0.48 \mathrm{~mm} ; 1.92 \pm 0.36 \mathrm{~mm})$ and after $(2.68 \pm 0.51 \mathrm{~mm}$; $1.87 \pm 0.43 \mathrm{~mm} ; 2.01 \pm 0.37 \mathrm{~mm})$, mean difference $-0.120 ;-0,085 ;-0.090 p=0.921$; 0.948 and 0.778 , respectively. Similarly, in the coronal section there are no significant changes in the right condylar angles before $\left(68.25 \pm 1.56^{\circ}\right)$ and after $\left(68.77 \pm 1.63^{\circ}\right)$ $p=0.217$; and left before $\left(68.92 \pm 1.63^{\circ}\right)$ and then $\left(69.30 \pm 2^{\circ}\right) p=0.215$. Conclusions. Sagittal osteotomy of the bilateral mandibular ramus in Class III patients is a surgical technique that offers minimal condylar alterations, since it maintains a condylar stability in the postoperative period at 9 months.

Keywords: Mandibular condyle; Cone-beam computed tomography; Orthognathic surgical procedures; Prognathism (source: MeSH NLM).

\section{Introducción}

La posición condilar tiene un papel importante en el sistema estomatognático, es el punto de partida para establecer y delimitar la relación céntrica (RC) ${ }^{1}$. Actualmente la RC es una relación maxilomandibular en la cual los cóndilos articulan con la porción avascular más delgada de sus respectivos discos con el complejo en la posición anterosuperior contra la inclinación de las eminencias articulares ${ }^{2}$. En Odontología, conocer esto es fundamental para poder identificar y tratar problemas en la articulación temporomandibular (ATM); en ortodoncia, su objetivo principal es lograr una armonía entre la RC y la oclusión céntrica, esta última se refiere a una posición dental interoclusal entre los dientes del maxilar y la mandíbula ${ }^{2,3}$.

Algunas investigaciones asocian signos y síntomas de problemas en la ATM con cambios posicionales de los cóndilos en la cavidad glenoidea ${ }^{4}$. Otros estudios mencionan que existen cambios en la posición condilar cuando se comparan sujetos que presentan Clase II y III esqueletal contra sujetos normales ${ }^{5}$. Los trabajos dirigidos al estudio de la posición condilar sugieren que la relación cóndilo-fosa más saludable y normal es aquella donde el cóndilo se sitúa centralizado antero-posteriormente dentro la fosa glenoidea ${ }^{6,7}$.

Las intervenciones quirúrgicas dentofaciales tienen como objetivo corregir discrepancias dentoesqueletales que puedan alterar la armonía facial, limitar la funcionalidad de la fonética, la deglución y en ocasiones llegan a interferir con la respiración con algún grado de apnea ${ }^{8,9}$. La osteotomía sagital bilateral de rama mandibular (OSBRM) ha sido la técnica más utilizada para la corrección de deformidades dentofaciales, su estabilidad continúa siendo un desafío para los cirujanos maxilofaciales. Fue Hullinhen en 1849 quien realizó la primer OSBRM para corregir una mala posición protusiva del segmento alveolar mandibular, seguido por Obwegeser y Trauner quienes en 1953 realizan juntos una intervención similar, fue hasta 1961 que Dalpont introduce una de las modificaciones más importantes realizadas sobre esta técnica, donde sugiere llevar el corte de la mandíbula hacia delante, por el cuerpo mandibular a la altura del primer y segundo molar donde desciende verticalmente hasta el borde basal. El cambio en el posicionamiento condilar juega un papel crítico en la estabilidad y recidiva de la cirugía ortognática por lo cual mantener al mínimo los cambios posicionales tras la intervención es el principal objetivo ${ }^{10-12}$.

El estudio de imagen para diagnóstico en la zona dentofacial tiene sus inicios con la implementación de imágenes bidimensionales, que en algunos casos brindan poca nitidez y calidad de imagen para poder diagnosticar cambios importantes en la posición condilar, ejemplo de ello son las radiografías panorámicas o lateral de cráneo. Actualmente se cuenta con la tomografía computarizada de haz cónico o TCHC (cone beam computed tomography, por sus siglas en ingles), que es una exposición tridimensional precisa, económica y de poca radiación que es ampliamente utilizada en la región maxilofacial; es el método principal para evaluar la posición y los cambios condilares en la articulación temporomandibular. Para los procedimientos de cirugía ortognática este estudio de imagen puede proporcionar mejor información del estado actual de las articulaciones temporomandibulares ${ }^{1,13}$. Por otra parte, es fundamental hacer un seguimiento y evaluar los posibles cambios que puedan presentarse en el plano oclusal y que puede verse comprometido, así como la aparición de afecciones condilares, tal es el caso de la resorción ósea del cóndilo tras una intervención quirúrgica de tal magnitud como lo es la OSBRM ${ }^{14,15}$. El propósito de este estudio fue comparar los cambios mediante la TCHC en el posicionamiento condilar en pacientes Clase III antes y después de la intervención de cirugía ortognática bimaxilar utilizando la OSBRM para la retroposición de la mandíbula.

\section{Métodos}

Se trata de una serie de casos clínicos, el grupo de estudio consistió en 30 pacientes con deformidad dentofacial Clase III no tratada que asistieron a consulta de diagnóstico en el periodo comprendido del año 2013 al 2016 en el Hospital Regional "General Ignacio Zaragoza" del Instituto de Seguridad y Servicios Sociales para los Trabajadores de la Ciudad de México. El tamaño de muestra se basó en el total de casos disponibles hasta el momento del estudio. 
Criterios de inclusión: pacientes clínicamente sanos sin síntomas de desórdenes en la ATM, sexo indistinto, sin intervenciones quirúrgicas ortognáticas previas, mayores de 15 años, sin trauma facial, con tratamiento ortodóncico previo de descompensación prequirúrgica, sin limitaciones en apertura y mayor a $40 \mathrm{~mm}$, con carta de consentimiento informado leída y firmada.

Criterios de exclusión: apertura bucal menor a $40 \mathrm{~mm}$ al momento de la cirugía, trauma facial, trismos o infección después de la intervención quirúrgica.

Todos los pacientes analizados fueron intervenidos por el mismo cirujano maxilofacial entre los martes y miércoles en un horario de 8 am a 2 pm, los estudios de imagen se realizaron con el aparato Galileos Comfort Plus Cone Beam 3D (Sirona, Dental System, Germany), mismo con que cuenta el servicio de cirugía maxilofacial del hospital.

Técnica quirúrgica. Todos los pacientes llenaron una historia clínica y se les realizó cirugía ortognática con osteotomía horizontal del maxilar tipo Lefort I ${ }^{16-18}$ en conjunto con la OSBRM para corregir la deformidad dentofacial. Se procedió a la osteosíntesis por medio de miniplacas de titanio del sistema 2,0 con tornillos monocorticales, el posicionamiento del cóndilo en la fosa glenoidea se efectuó realizando presión sobre el ángulo mandibular en dirección superior y posterior, manteniendo continuidad en el borde basal; paso seguido el cirujano procede a colocar la placa de osteosíntesis, todos los pacientes presentaron una Clase I esquelética al término de la cirugía. Como maniobras a destacar es la utilización de una guía quirúrgica final, donde el paciente la utiliza por una semana con boca cerrada mediante elásticos de tracción, esto para ayudar a una recuperación menos molesta.

Especificaciones del estudio de imagen. Para el equipo de TCHC las especificaciones son las siguientes: un volumen esférico y diámetro de $15,4 \mathrm{~cm}$, voltaje del tubo de $98 \mathrm{Kv}$, amperaje de 3 a $6 \mathrm{~mA}$, tiempo de escaneo de 14 segundos.

Análisis de la posición condilar. Este análisis se realizó por medio de tres tomas con la TCHC, siendo la primera prequirúrgica (2-4 días previos), seguida de una toma a los 4 días posquirúrgicos y una última a los 9 meses posteriores a la intervención, en todas ellas se colocó al paciente en relación céntrica, con la cabeza en plano de Frankfort horizontal al piso, la manipulación de este equipo se llevó a cabo por personal del hospital capacitado, y fue el mismo en todas y cada una de las mediciones entre los pacientes.

Mediciones de la posición condilar. En este estudio se analizaron dos variables de interés en dos tiempos (prequirúrgico y posquirúrgico), en primer plano el espacio cóndilo-fosa en un corte sagital en una situación anterior, craneal y posterior (Figura 1); en segundo plano la angulación de los cóndilos según el método modificado de Kim y cols. ${ }^{19}$ el cual toma como referencia el límite

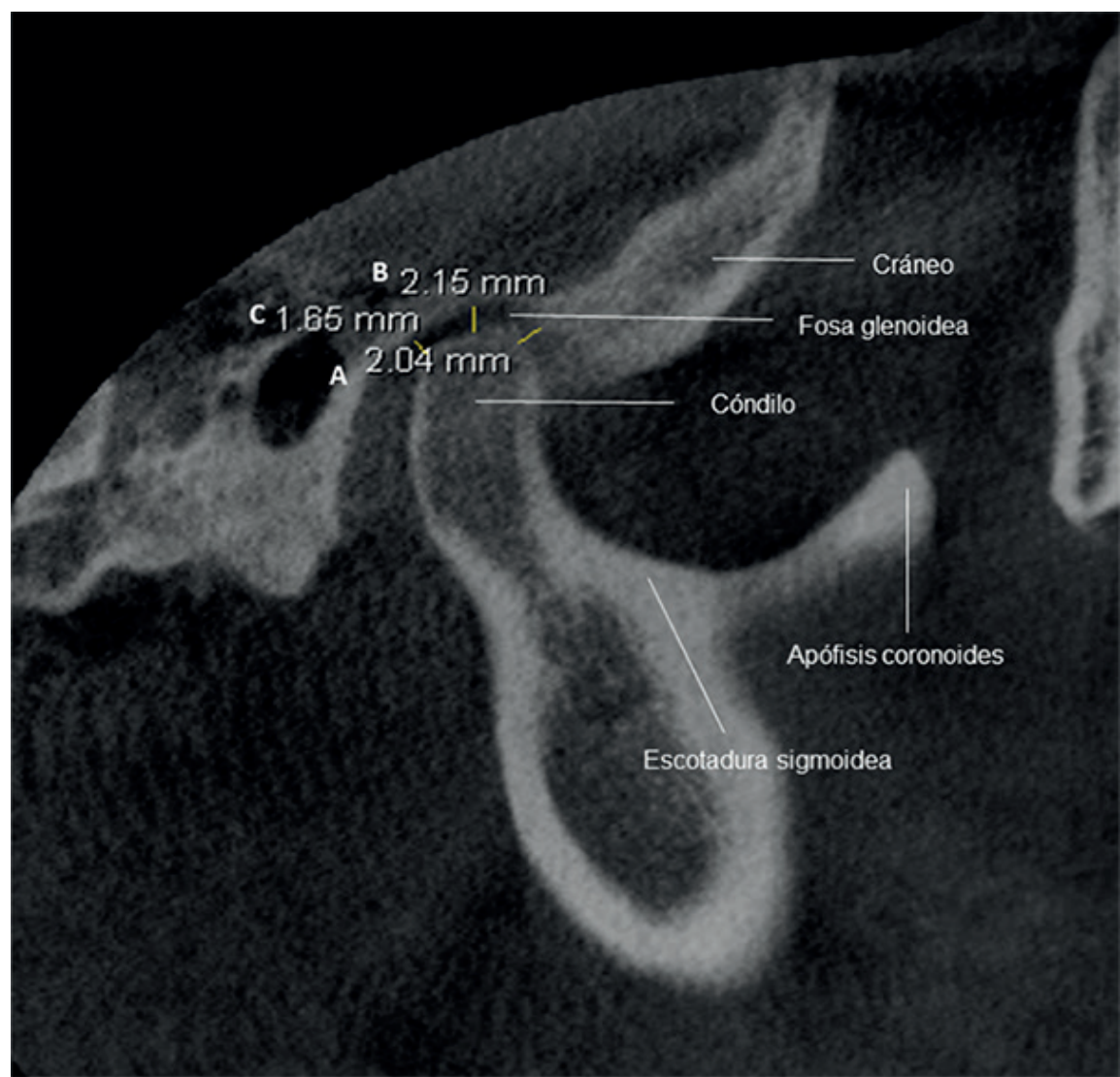

Figura 1. Medición de la posición condilar por medio del espacio entre el cóndilo y la fosa glenoidea en un corte sagital. Situación anterior (A), craneal (B) y posterior (C) 
inferior del agujero magno, el punto medio condilar y el borde anterior de la rama mandibular en un corte coronal (Figura 2).

Este estudio fue avalado por el departamento de enseñanza del Hospital Regional "General Ignacio Zaragoza” (CDMX,México), y se siguieron los lineamientos éticos de la Norma Oficial Mexicana NOM-012-SSA3-2012, para protocolos de investigación en seres humanos y de la Norma Oficial Mexicana NOM-004-SSA3-2012, para el uso y confidencialidad el expediente clínico.

Para el procesamiento de los datos se creó una base de datos digital en formato Excel de Microsoft ${ }^{\circledR}$, mientras que para el análisis de los mismos se empleó el progra- ma estadístico de libre distribución R Studio, 2019. La prueba estadística que se empleo fue ANOVA de un factor y se trabajó a un nivel de significancia del 5\%.

\section{Resultados}

En el grupo de estudio la distribución por sexo fue de 16 mujeres y 14 hombres, con un rango de edad de 15 a 40 años y una media de 28,1 años. En este análisis se realizaron mediciones prequirúrgicas en el espacio cóndilo-fosa, tanto para el lado derecho como el izquierdo en mujeres y hombres, y la medición promedio del grupo total, la unidad de medida es en milímetros y se muestra la media y desviación estándar, ver Tabla 1.

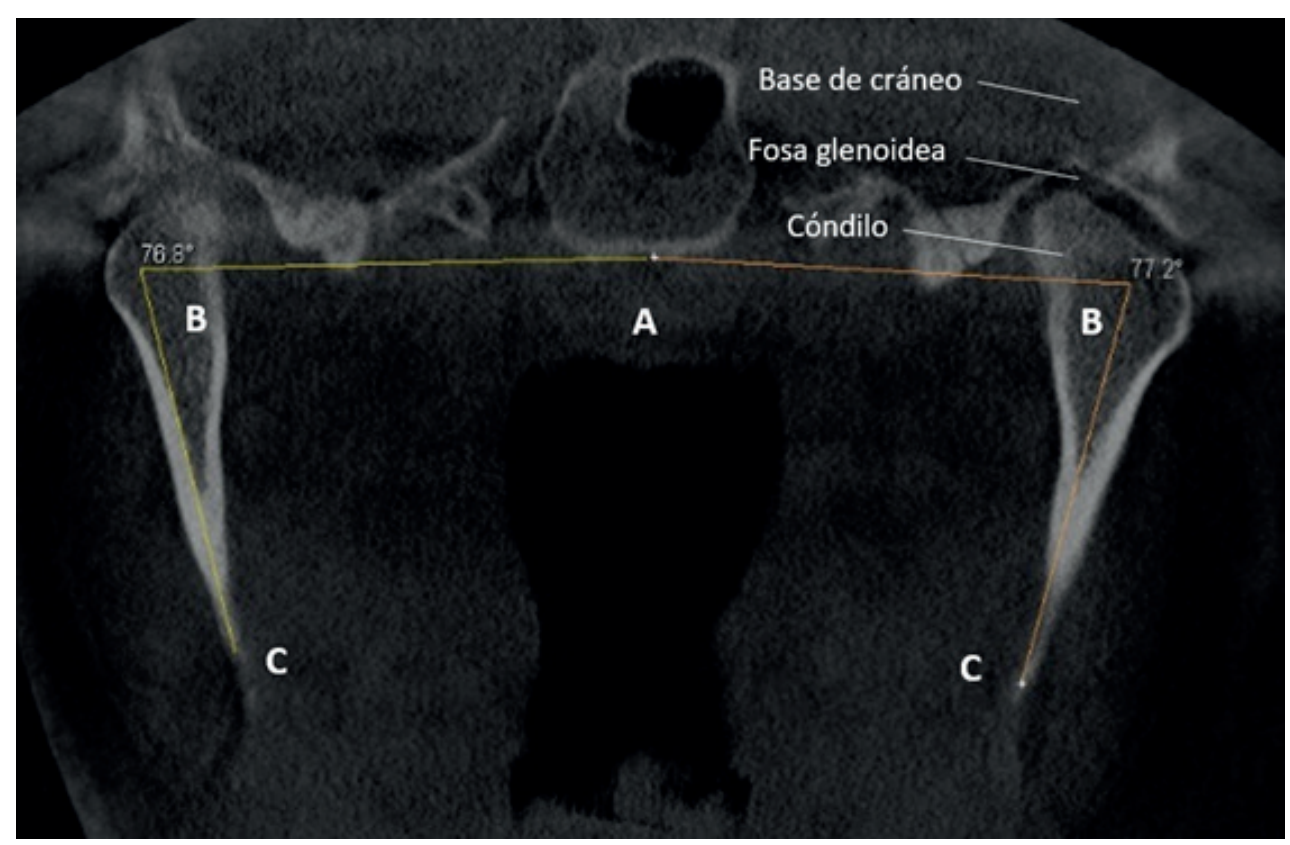

Figura 2. Medición de la angulación condilar por el método de Kim y cols. ${ }^{19}$ en un corte coronal. Límite inferior de agujero magno (A), ángulo del punto medio condilar (B) y borde anterior de la rama mandibular (C)

Tabla 1. Medición prequirúrgica de los espacios del cóndilo-fosa de lado derecho e izquierdo en mujeres y hombres

\begin{tabular}{|c|c|c|}
\hline $\begin{array}{l}\text { Mujeres } \\
n=16\end{array}$ & $\begin{array}{l}\text { Lado derecho } \\
\text { (Prequirúrgica) }\end{array}$ & $\begin{array}{l}\text { Lado izquierdo } \\
\text { (Prequirúrgica) }\end{array}$ \\
\hline \multicolumn{3}{|l|}{ Variables } \\
\hline EA & $2,81 \pm 0,51$ & $2,53 \pm 0,58$ \\
\hline $\mathrm{EC}$ & $1,53 \pm 0,16$ & $2,04 \pm 0,59$ \\
\hline $\mathrm{EP}$ & $1,74 \pm 0,29$ & $2,08 \pm 0,32$ \\
\hline \multicolumn{3}{|l|}{$\begin{array}{l}\text { Hombres } \\
n=14\end{array}$} \\
\hline \multicolumn{3}{|l|}{ Variables } \\
\hline EA & $2,75 \pm 0,74$ & $2,12 \pm 0,43$ \\
\hline EC & $1,71 \pm 0,33$ & $1,90 \pm 0,41$ \\
\hline $\mathrm{EP}$ & $1,95 \pm 0,36$ & $1,87 \pm 0,59$ \\
\hline $\begin{array}{l}\text { Total } \\
\mathrm{n}=30\end{array}$ & \multicolumn{2}{|c|}{ (Prequirúrgica) } \\
\hline Variables & \multicolumn{2}{|c|}{ Ambos lados } \\
\hline EA & \multicolumn{2}{|c|}{$2,56 \pm 0,55$} \\
\hline $\mathrm{EC}$ & \multicolumn{2}{|c|}{$1,78 \pm 0,48$} \\
\hline $\mathrm{EP}$ & \multicolumn{2}{|c|}{$1,92 \pm 0,36$} \\
\hline
\end{tabular}

EA: Espacio anterior, EC: Espacio craneal y EP: Espacio posterior 
En el corte coronal se evaluó la medición sobre la angulación condilar prequirúrgica se obtuvo un valor inicial de $68,25^{\circ}$ en el lado derecho y de $68,92^{\circ}$ respectivamente.

Una vez efectuadas las intervenciones quirúrgicas se procedió a realizar las mediciones para evaluar la posición condilar mediante la TCHC en corte sagital y coronal a los 4 días y 9 meses posteriores a la cirugía. Al efectuar el análisis estadístico para determinar si existían diferencias entre la posición condilar inicial (prequirúrgica) y final (posquirúrgica) no se encontraron diferencias significativas (Tabla 2).

Se realizó la evaluación de la angulación condilar entre la etapa prequirúrgica y posquirúrgica tanto de lado derecho como izquierdo (Tabla 3 ).

\section{Discusión}

La corrección de las deformidades dentofaciales tiene como desafío la alta recidiva en las intervenciones de cirugía ortognática, existen factores que condicionan la estabilidad y la recidiva, entre los cuales se menciona a la extensión del movimiento, el tipo y materiales de fijación, el plano del ángulo mandibular, el control del segmento proximal, la cantidad tejido blando, la tensión muscular, la falta de crecimiento, el grado de remodelación, la edad preoperatoria y la habilidad del cirujano, mismos que contribuyen a una inadecuada posición e inestabilidad condilar ${ }^{19-21}$.En nuestra experiencia, no tuvimos recidiva en la población estudiada en un con- trol posoperatorio a los 9 meses, se destaca la consistencia en el abordaje por parte del cirujano y la mínima modificación del espacio condilar.

Los reportes en la literatura científica coinciden en que la resonancia magnética en la actualidad continúa siendo un estudio ideal para valorar estructuras óseas y de igual manera la articulación temporomandibular, sin embargo, su alto costo es una desventaja frente al TCHC, donde este último permite un análisis detallado para medir espacios diminutos y complejos como lo es la zona condilar. Para obtener imágenes fiables de las estructuras craneomandibulares se decidió utilizar el TCHC en mediciones prequirúrgicas, posquirúrgicas inmediatas a 4 días y a 9 meses posquirúrgicos; con esto se logró realizar la evaluación de la posición condilar a detalle.

La articulación temporomandibular posee una anatomía compleja y dinámica, donde un manejo correcto y preciso de los puntos de referencia son cruciales cuando de medición se trata ${ }^{20,22}$. En nuestro estudio los puntos anatómicos de referencia son claramente visualizados, y esto permite identificar pequeños datos de desviación.

En cuanto a la medición del retroceso mandibular Kim y cols. ${ }^{19}$, indican en su estudio movimientos de $7,4 \mathrm{~mm}$, Draenert y cols. ${ }^{20}$, mencionan que no existen cambios significativos, mostrando un valor $\mathrm{p}$ de 0,69 en el espacio intercondilar y de 0,27 para la angulación intercondilar; en nuestros resultados observamos para la angulación condilar en corte sagital valores $p$ de 0,217

Tabla 2. Cambios en el espacio interarticular en corte sagital de lado derecho e izquierdo en diferentes tiempos de intervención

\begin{tabular}{ccccc}
\hline Variables & Medida inicial (Prequirúrgica) & $\begin{array}{c}\text { Medida intermedia (4 días } \\
\text { posquirúrgica) }\end{array}$ & $\begin{array}{c}\text { Medida final } \\
\text { (9 meses posquirúrgica) }\end{array}$ & Valor de $\mathbf{p}$ \\
\hline EAD & $2,78 \pm 0,47$ & $3,03 \pm 0,48$ & $2,88 \pm 0,46$ & 0,135 \\
ECD & $1,61 \pm 0,26$ & $1,75 \pm 0,25$ & $1,68 \pm 0,26$ & 0,133 \\
EPD & $1,84 \pm 0,34$ & $2,02 \pm 0,38$ & $1,92 \pm 0,37$ & 0,152 \\
EAI & $2,34 \pm 0,54$ & $2,57 \pm 0,65$ & $2,48 \pm 0,56$ & 0,324 \\
ECI & $1,96 \pm 0,58$ & $2,17 \pm 0,63$ & $2,06 \pm 0,60$ & 0,413 \\
EPI & $2,00 \pm 0,37$ & $2,22 \pm 0,46$ & $2,10 \pm 0,38$ & 0,110 \\
\hline
\end{tabular}

EAD: Espacio anterior derecho, ECD: Espacio craneal derecho, EPD: Espacio posterior derecho, EAI: Espacio anterior izquierdo, ECl: Espacio craneal izquierdo, EPI: Espacio posterior izquierdo.

Los resultados de las medidas se presentan como media y desviación estándar.

La unidad de medida es en milímetros.

Prueba ANOVA de un factor

Tabla 3. Cambios en la angulación condilar de lado derecho e izquierdo en un corte coronal en diferentes tiempos de intervención

\begin{tabular}{cccc}
\hline Variables & $\begin{array}{c}\text { Medida inicial } \\
\text { (Prequirúrgica) }\end{array}$ & $\begin{array}{c}\text { Medida intermedia (4 días } \\
\text { posquirúrgica) }\end{array}$ & $\begin{array}{c}\text { Medida final } \\
\text { (9 meses posquirúrgica) }\end{array}$ \\
\hline ACD & $68,25 \pm 1,56$ & $68,96 \pm 1,61$ & $68,77 \pm 1,63$ \\
ACI & $68,92 \pm 1,63$ & $69,73 \pm 1,68$ & 6,217 \\
\hline
\end{tabular}

ACD: Angulo condilar derecho, $\mathrm{ACl}$ : Angulo condilar izquierdo.

Los resultados de las medidas se presentan como media y desviación estándar.

La unidad de medida es en grados.

Prueba ANOVA de un factor 
y 0,215 para lado derecho e izquierdo respectivamente. Del mismo modo, Kim y cols. ${ }^{19}$ indican que existe un cambio significativo en el espacio articular anterior derecho e izquierdo, con valor de p de 0,01 y 0,04 respectivamente.

Byung-Joon Choi y cols. ${ }^{23}$, en su estudio, reportan que no se encuentran cambios significativos en la evaluación de la posición condilar. En nuestra experiencia no se encontraron cambios significativos entre la medición condilar inicial y la medición final. Esto sugiere que la OSBRM aunado a la técnica de reposición condilar y la fijacion de los segmentos óseos posterior a la osteotomía ofrece confiabilidad para llevarlo a cabo sin alterar la adaptación condilar previa de los pacientes sin datos de trastornos temporomandibulares.

Aún existe controversia si la relación cóndilo-fosa de la articulación temporomandibular en céntrica está asociado a trastornos temporomandibulares, por lo que es necesario fortalecer y estandarizar los protocolos diagnósticos y terapéuticos en este tema ${ }^{24}$.

La intervención con osteotomía sagital bilateral de rama mandibular en pacientes Clase III es una técnica quirúrgica que ofrece mínimas alteraciones condilares, pues mantiene una estabilidad condilar en el posoperatorio a 9 meses, lo que se traduce a una posición estable y funcional. Son necesarios más estudios a largo plazo sobre la posición condilar posterior a la intervención quirúrgica ortognática para un mejor entendimiento y manejo del impacto funcional resultante.

\section{Agradecimientos}

Los autores agradecen al Director y personal del Hospital Regional "General Ignacio Zaragoza”, ISSSTE, de la CDMX por permitir realizar el estudio a través de su infraestructura y equipos. Así como reconocemos la inigualable labor del Jefe de Cirugía Maxilofacial, Dr. Ernesto Miranda Villasana.

\section{Referencias bibliográficas}

1. Dalili Z, Khaki N, Kia SJ, Salamat F. Assessing joint space and condylar position in the people with normal function of temporomandibular joint with cone-beam computed tomography. J Dent Res. 2012;9(5):607-12. DOI: $10.4103 / 1735-3327.104881$

2. Rinchuse DJ, Kandasamy S. Centric relation: a historical and contemporary orthodontic perspective. J Am Dent Assoc. 2006;137(4):494-501. DOI: 10.14219/jada.archive.2006.0222

3. Hidaka O, AdachI S, Takada K. The difference in condylar position between centric relation and centric occlusion in pretreatment Japanese orthodontic patients. Angle Orthod. 2002;72(4):295-301. DOI: 10.1043/0003-3219(2002)072<0295:tdicpb>2.0.co;2

4. Ren YF, Isberg A, Westesson PL. Condyle position in the temporomandibular joint: comparison between asymptomatic volunteers with normal disk position and patients with disk displacement. Oral Surg Oral Med Oral Pathol Oral Radiol Endod. 1995;80(1):101-107. DOI: 10.1016/S1079-2104(95)80025-5
5. Fraga MR, Rodrigues AF, Ribeiro LC, da Silva Campos MJ, Vitral RWF. Anteroposterior condylar position: A comparative study between subjects with normal occlusion and patients with Class I, Class II Division 1, and Class III malocclusions. 2013;19:903. DOI: 10.12659/ MSM.889528.

6. Blaschke DD, Blaschke TJ. Normal TMJ bony relationships in centric occlusion. J Dent Res. 1981;60(2):98104. DOI: $10.1016 / 0022-3913(85) 90029-0$

7. Pullinger AG, Hollender L, Solberg WK, Petersson A. A tomographic study of mandibular condyle position in an asymptomatic population. J Prosthet Dent. 1985;53(5):706-713.

8. Faria Patrícia Toledo Monteiro, Ruellas Antonio Carlos de Oliveira, Matsumoto Mírian Aiko Nakane, Anselmo-Lima Wilma T., Pereira Fabiana C. Dentofacial morphology of mouth breathing children. Braz Dent J. 2002;13(2):129-132. DOI: 10.1590/S010364402002000200010 .

9. Basheer B, Hegde KS, Bhat SS, Umar D, Baroudi K. Influence of mouth breathing on the dentofacial growth of children: a cephalometric study. J Int Oral Health. 2014;6(6):50-55.

10. Obwegeser $\mathrm{H}$. The indications for surgical correction of mandibular deformity by the sagittal splitting technique. Br J Oral Surg. 1963;1:157-171. DOI: 10.1016/S0007117X(63)80067-0

11. Hunsuck EE. A modified intraoral sagittal splitting technic for correction of mandibular prognathism. Oral Surg. 1968;26:49-52.

12. Will LA, Joondeph DR, Hohl TH, West RA. Condylar position following mandibular advancement: its relationship to relapse. J Oral Maxillofac Surg .1984;42(9):578588. DOI: $10.1016 / 0278-2391(84) 90088-0$

13. Roque-Torres Gina D, Meneses-López Abraham, Norberto Bóscolo Frab, De Almeida Solange María, Haiter Neto Francisco. La tomografía computarizada cone beam en la ortodoncia, ortopedia facial y funcional. Rev Estomatol Herediana. 2015;25(1):61-78.

14. Olate S, Netto C \& de Miranda H D. Manipulación del plano oclusal en cirugía ortognática: consideraciones faciales. Int J Odontostomat. 2010;4(1):23-32.

15. López EO \& Jaramillo PU. Resorción Condilar Idiopática después de Cirugía Ortognática Bimaxilar. OdontoInvestigación. 2019;5(1).

16. Reyneke JP, Ferretti C. Intraoperative diagnosis of condylar sag after bilateral sagittal split ramus osteotomy. Br J Oral Maxillofac Surg. 2002;40(4):285-292. DOI: 10.1016/S0266-4356(02)00147-X

17. Ueki K, Marukawa K, Nakagawa K, Yamamoto E. Condylar and temporomandibular joint disc positions after mandibular osteotomy for prognathism. J. Oral Maxillofac Surg. 2002;60(12):1424-1432. DOI: 10.1053/ joms.2002.36098

18. Ueki K, Marukawa K, Shimada M, Nakagawa K, Yamamoto E. Change in condylar long axis and skeletal stability following sagittal split ramus osteotomy and intraoral vertical ramus osteotomy for mandibular prognathia. 
J Oral Maxillofac Surg. 2005;63(10):1494-1499. DOI: 10.1016/j.joms.2005.06.013

19. Kim YI, Cho BH, Jung YH, Son WS, Park SB. Cone-beam computerized tomography evaluation of condylar changes and stability following two-jaw surgery: Le Fort I osteotomy and mandibular setback surgery with rigid fixation. Oral Surg Oral Med Oral Pathol Oral Radiol Endod. 2011;111(6):681-687. DOI: 10.1016/j. tripleo.2010.08.001.

20. Draenert FG, Erbe C, Zenglein V, Kämmerer PW, Wriedt S, Al Nawas B. 3D analysis of condylar position after sagittal split osteotomy of the mandible in mono-and bimaxillary orthognathic surgery-a methodology study in 18 patients. J Orofac Orthop. 2010;71(6):421-429. DOI: $10.1007 / \mathrm{s} 00056-010-1021-9$

21. Seeberger R, Thiele OC, Mertens C, Hoffmann J, Engel M. Proximal segment positioning with high oblique sagittal split osteotomy: indications and limits of intraoperative mobile cone-beam computerized tomography. Oral Surg Oral Med Oral Pathol Oral Radiol. 2013;115(6):731-736. DOI: 10.1016/j. oooo.2012.10.016
22. Lukat TD, Perschbacher SE, Pharoah MJ, Lam EW. The effects of voxel size on cone beam computed tomography images of the temporomandibular joints. Oral Surg Oral Med Oral Pathol Oral Radiol. 2015;119(2):229-237. DOI: 10.1016/j.oooo.2014.10.015.

23. Choi BJ, Kim BS, Lim JM, Jung J, Lee JW, Ohe JY. Positional change in mandibular condyle in facial asymmetric patients after orthognathic surgery: cone-beam computed tomography study. Maxillofac Plast Reconstr Surg. 2018;40(1):13. DOI:10.1186/s40902-018-0152-6

24. Reiter S, Goldsmith C, Emodi-Perlman A, Friedman-Rubin P, Winocur E. Masticatory muscle disorders diagnostic criteria: the American Academy of Orofacial Pain versus the research diagnostic criteria/temporomandibular disorders (RDC/TMD). J Oral Rehabilitation. 2012;39:941-947. 
